\title{
Observation of temporal evolution following laser triggered rf breakdown in vacuum
}

\author{
Jiahang Shao, ${ }^{1,2, *}$ Huaibi Chen, ${ }^{1}$ Yingchao Du, ${ }^{1}$ Wei Gai, ${ }^{2}$ Wenhui Huang,,${ }^{1}$ Chunguang Jing, $, 3, \dagger$ \\ Jiaru Shi, ${ }^{1}$ Chuanxiang Tang, ${ }^{1}$ Faya Wang, ${ }^{4}$ and Lixin Yan $^{1}$ \\ ${ }^{1}$ Department of Engineering Physics, Tsinghua University, Beijing 100084, People's Republic of China \\ ${ }^{2}$ Argonne National Laboratory, Lemont, Illinois 60439, USA \\ ${ }^{3}$ Euclid Techlabs, Solon, Ohio 44139, USA \\ ${ }^{4}$ SLAC National Accelerator Laboratory, Menlo Park, California 94025, USA
}

(Received 27 February 2014; published 21 July 2014)

\begin{abstract}
Radio frequency breakdown is one of the fundamental phenomena that limits the operational performance of most of high power and high gradient vacuum $\mathrm{rf}$ devices. We report on experimental results of rf breakdown in an $S$-band photocathode gun triggered by an intensity controlled laser. Through measurement and analysis of the time dependence of the collected current at the gun exit and the stored $\mathrm{rf}$ energy in the cavity, one can gain insight into the time evolution of the rf breakdown process. Multiple breakdowns were observed within one rf pulse due to power flow between cells after the initial emission. Similarities of the laser-triggered breakdowns to those occurring in the course of cavity conditioning and normal operation are found by comparing the postbreakdown signals in both cases. It is shown that an intense laser can offer a more controllable and flexible method for rf breakdown studies.
\end{abstract}

DOI: 10.1103/PhysRevSTAB.17.072002

PACS numbers: 29.20.Ej, 52.80.Pi, 79.70.+q

\section{INTRODUCTION}

The ultimate performance of high power and high gradient metallic vacuum of devices is limited primarily by rf breakdowns, which occur on the surface of vacuum metallic structures. Radio frequency breakdown is a very complicated physical phenomenon that may involve localized field emission, eddy current, plasma formation, rf power flow, pulse heating, metal fatigue, crystallographic defects, and so on [1-5]. Despite previous extensive experimental and theoretical efforts, the nature of $\mathrm{rf}$ breakdown appears to be largely unpredictable leading to difficulties in conducting a systematic and conclusive investigation. One approach to the study of $\mathrm{rf}$ breakdown is to accumulate breakdown events for tens of millions of input rf pulses so that the statistical behavior of different structures can be compared [6-11]. Direct-current breakdown experiments provide a flexible and affordable approach for breakdown study [12,13]; however, the relation of the physics of dc breakdown to rf breakdown is not clear. In this article, we present a controllable way to study the evolution of individual rf breakdown events over a time scale of nanoseconds using an intense laser to trigger breakdowns in an rf structure. By this method, the spatial and temporal occurrence of a breakdown can be precisely

\footnotetext{
*shaojh07@mails.tsinghua.edu.cn

†jingchg@anl.gov
}

Published by the American Physical Society under the terms of the Creative Commons Attribution 3.0 License. Further distribution of this work must maintain attribution to the author(s) and the published article's title, journal citation, and DOI. controlled by the trigger laser so that we are able to efficiently record and examine signals of explosive electron emission (EEE) and electric field variation as the consequence of rf breakdown. This study is closely linked to $\mathrm{rf}$ breakdown in high gradient particle accelerating structures, and may also provide useful information for breakdown in other metallic high power if devices.

A high accelerating gradient is one of the main considerations in the design of a compact linear collider [14]. Through the use of modern technologies like optimizing the structure geometry using electromagnetic computer aided design tools, highly accurate surface machining and finishing, improved surface preparation, etc., the gradient of normal conducting accelerators has been improved dramatically in the past few decades. Recently, a $120 \mathrm{MV} / \mathrm{m}$ gradient was reached in a The Compact Linear Collider $X$-band test structure with a $252 \mathrm{~ns}$ pulse length and breakdown rate (BDR) around $10^{-6} /$ pulse $/ \mathrm{m}$ [6]. An $X$-band single cell structure achieved $160 \mathrm{MV} / \mathrm{m}$ gradient with a $150 \mathrm{~ns}$ pulse length and BDR of $10^{-3} / \mathrm{pulse} / \mathrm{m}$ [7]. However, at this point $\mathrm{rf}$ breakdown limits the structures from achieving higher gradient operation with a reasonable pulse length ( $>200 \mathrm{~ns}$ for $X$-band).

The process of laser-triggered if breakdown is always accompanied with EEE [15-17]. The EEE mechanism is the rapid eruption of a large electron current on a metallic surface where a plasma sheath is formed. Commonly this localized plasma is initiated by ionization of metal vapor due to the local heating from field emission [18]. The current density in EEE is capable of reaching $1 \mathrm{kA} / \mathrm{cm}^{2}$ so that it is widely used as a cathode of gigawatt class $\mathrm{rf}$ 
generators $[19,20]$. Through experimental comparison of rf breakdown with and without the laser trigger, we confirmed that EEE was the consequence of vacuum rf breakdown on the metal surface. The observed duration of the electron eruption in the experiment is around $100 \mathrm{~ns}$ (which may depend on the rf frequency) with the same current density level, but the concurrent plasma needs a much longer time to decay for the process of the plasma expansion and recombination to occur so that the entire rf pulse may continue to be blocked for a period as long as microseconds (as observed in our experiment and other experiments [21]).

This paper is organized as follows: Section II presents the experimental setup. The experimental results and the associated simulation analysis are provided in Sec. III. Some experimental observation as well as advantages of the laser trigger method for $r f$ breakdown studies are discussed in Sec. IV. Finally, a summary of results along with future directions of this research are given in Sec. V.

\section{EXPERIMENT SETUP}

The laser-triggered rf breakdown experiment was conducted at one of the $S$-band photocathode gun beam lines at the Accelerator Laboratory of Tsinghua University [22].

The 1.6-cell gun operates at $2856 \mathrm{MHz} \pi$ mode with an $\mathrm{rf}$ pulse length of $6 \mu \mathrm{s}$, rise time of $2 \mu \mathrm{s}$, and fall time of $2 \mu \mathrm{s}$. The cathode surface of the gun is a solid, demountable copper plate. With 4.8 MW input power, the available rf field on the central area of the cathode surface can be as large as $58 \mathrm{MV} / \mathrm{m}$. In normal operation a UV laser (wavelength $266 \mathrm{~nm}$, pulse width $1.2 \mathrm{ps}$ full width at half maximum, $1 \mathrm{~mm}^{2}$ spot size on the cathode, and $2 \mathrm{~mJ}$ maximum energy) is directed onto the central diamond polished area of the cathode to generate photoelectron emission. During the laser trigger breakdown experiment, the laser was directed at the cathode $\sim 0.5 \mu$ s before the end of the flat top when the cavity had been fully filled. The mirror inside the vacuum chamber was remotely controlled so that the breakdown location triggered by the laser could be changed. (Up to an offset of $1.5 \mathrm{~mm}$ in the experiment, the on-axis field varied less than $0.2 \%$.)

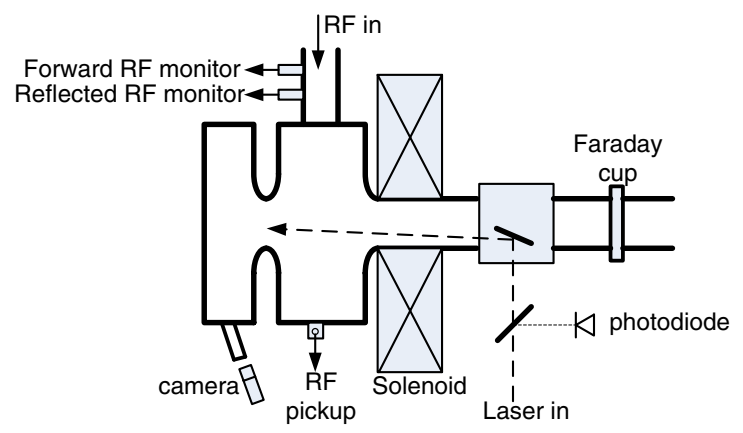

FIG. 1. Setup of the laser-triggered rf breakdown experiments at Tsinghua University.
A schematic layout of the experiment is shown in Fig. 1. Diagnostics involved in the experiment were a bidirectional coupler to monitor the input and reflected $\mathrm{rf}$ signals, a loop antenna (pickup) in the full cell to monitor the rf signal inside the cavity, a Faraday cup outside the gun to measure the beam current during breakdown events, and a CCD camera to monitor and index the breakdown locations through the viewport at the half cell. All signals were recorded using a $50 \mathrm{Gs} / \mathrm{s}$ sampling rate, $12 \mathrm{GHz}$ bandwidth digital scope synchronized to the incoming laser signal or to the input rf signal when the laser pulse was absent.

\section{EXPERIMENT RESULTS}

\section{A. Normal operation without breakdown}

In normal operation of the photocathode gun, a laser pulse with energy less than $5 \mu \mathrm{J}$ is used to generate a $\sim 100$ pC photoelectron bunch when the gun is fully filled. Figure 2 shows the typical recorded signals including the rectified waveforms of input and reflected rf pulses in Fig. 2(a), the rectified rf pickup signal in Fig. 2(b), and the Faraday cup signal excited by the emitted photoelectrons in
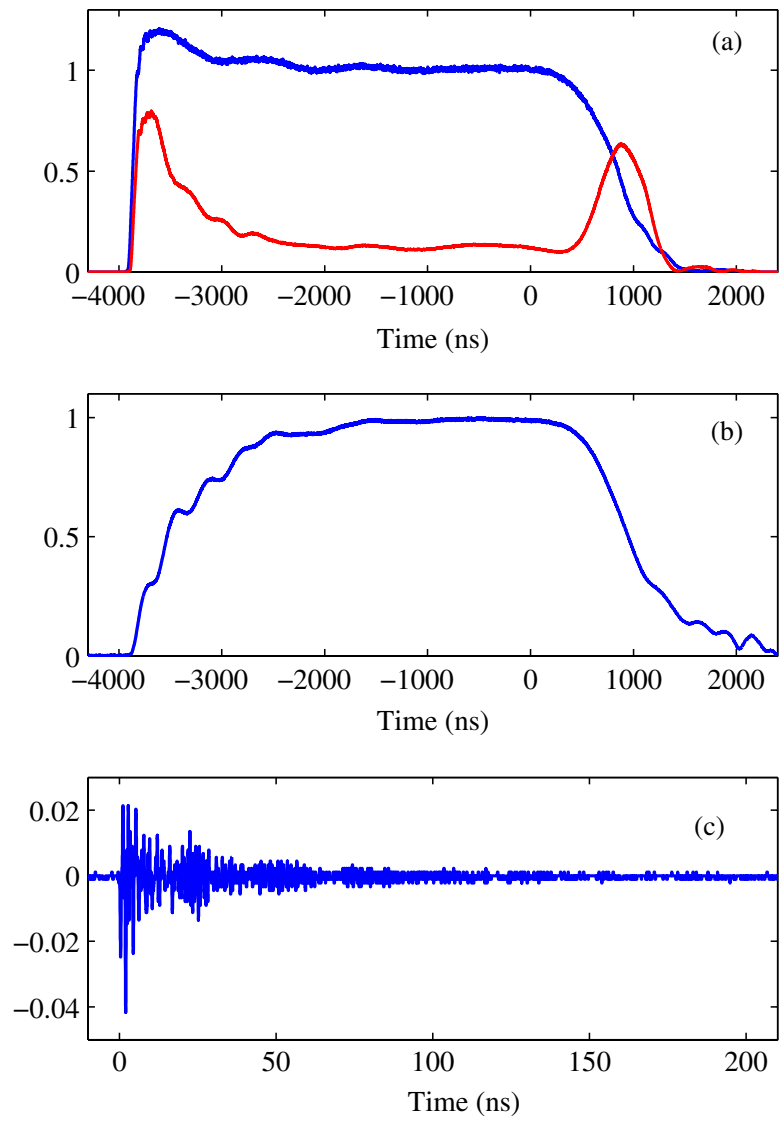

FIG. 2. Typical waveforms without breakdown. (a) Envelopes of the normalized input (blue line) and reflected (red line) rf pulse. (b) Envelope of the normalized pickup signal. (c) The Faraday cup signal in amperes as excited by photoelectrons. 
Fig. 2(c). The origin of the time axis $(t=0)$ has been set to the moment when the incident laser hits the cathode.

The duration of the photoelectron current is several picoseconds and the Faraday cup signal appears to be a damped oscillation lasting tens of nanoseconds because of ringing in the data acquisition system. It contains the system transfer function of the Faraday cup which can be extracted later to rebuild the time structure of breakdown currents. Details will be discussed in the following subsections.

\section{B. Radio frequency breakdown without a laser trigger}

Like other high power rf devices, the rf breakdown may occasionally occur when the photocathode gun is pushed to its maximum operational gradient, even without the laser. During the experiment, at the gradient of $58 \mathrm{MV} / \mathrm{m}$, tens of rf breakdown events were recorded over hundreds of thousands of rf pulses. When a breakdown happened, spikes and sequential oscillation could be observed in the envelope of the pickup signal. Similar spikes were also found in the refection signal. A significant current was captured by the Faraday cup. All the recorded breakdowns without laser triggers can be classified into two types according to the breakdown current profile captured by the Faraday cup. For the single-breakdown type, there was only one current pulse; while for the multiple breakdown type, two or more pulses could be detected within an interval of $200 \mathrm{~ns}$. Typical recorded waveforms are shown in Figs. 3(a) and 3(b).

These spikes are caused by the excitation of higher order modes (HOM) of the gun. The breakdown current serves as another source other than the original input rf power from klystron. The current lasts for tens of nanoseconds and contains a bunch train of captured electrons separated by one rf cycle of the fundamental mode. Each bunchlet of electrons comes into phase with the rf wave after the initial acceleration in the half cell and the duration is tens of picoseconds (from simulation using the PARMELA code [23]). Thus HOMs of the cavity can be excited by the short bunchlets. In particular, HOMs whose frequency is close to a harmonic of the fundamental mode can be coherently reinforced by the bunch train.

In order to study the evolution of the fundamental mode and HOMs during rf breakdown events, a short time fast Fourier transformation (FFT) is applied along the recorded pickup signal with a $10 \mathrm{~ns}$ window moving at $0.5 \mathrm{~ns}$ per step. Results in Figs. 3(c) and 3(d) clearly show the excitation of a $8.6 \mathrm{GHz} \mathrm{HOM}$ (close to the third harmonic of $2.856 \mathrm{GHz}$ ) associated with the collapse of the fundamental mode. Simulation by CST Microwave Studio [24] confirms this HOM to be the $\mathrm{TM}_{410}$ mode of the gun. Multiple azimuthal variations of the EM pattern of the HOM are caused by the large divergence of emitted electrons. The onset of a breakdown can then be defined at the moment of excitation of the $8.6 \mathrm{GHz} \mathrm{HOM}$. Among
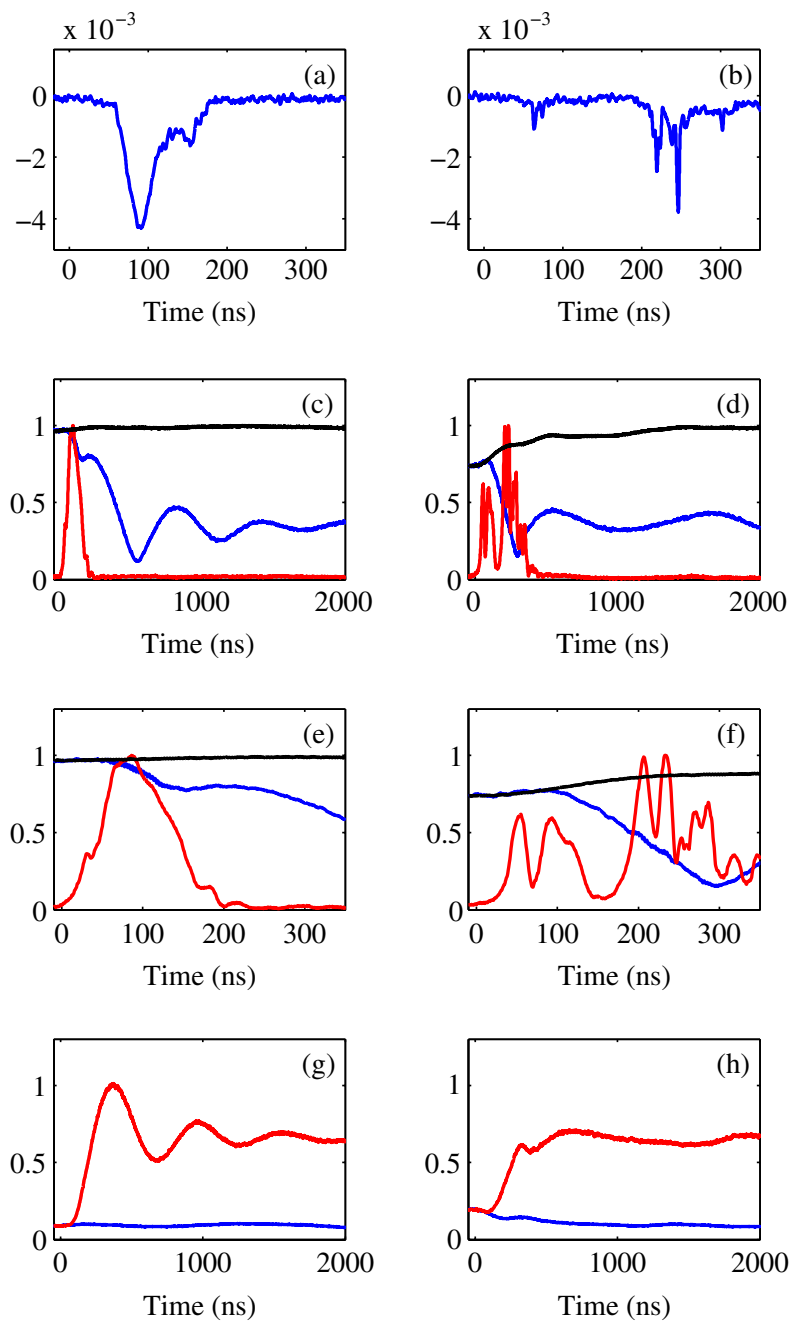

FIG. 3. Radio frequency breakdown without laser trigger of single-breakdown event (left) and multiple-breakdown event (right). (a),(b) The Faraday cup signal in Amperes. (c),(d) Normalized FFT amplitude of the pickup signal in different frequency bands as a function of time, including the fundamental mode without breakdown (black line), the fundamental mode with breakdown (blue line), and the $8.6 \mathrm{GHz} \mathrm{HOM}$ with breakdown (red line). (e),(f) Same signals in (c),(d) with a zoom-in view around 0 ns. (g),(h) Normalized FFT amplitude of the reflection signal, including the fundamental mode without breakdown (blue line) and with breakdown (red line).

the recorded data, the onset of a breakdown is located randomly within the entire rf pulse (including the rise and fall times). We also observed a 40-140 ns time delay between the start of the collapse of the fundamental mode and the excitation of the HOM [shown in Figs. 3(e) and 3(f)]. The FFT method is also applied to the reflection signal. Results in Figs. 3(g) and 3(h) show the increased reflected power during breakdown events.

The time delay is because the breakdown location is in the half cell and away from the axis. Under this condition, only the field in the half cell collapses immediately after the onset of breakdown and it takes a certain period of time for 
the full cell to respond to the energy imbalance between two cells. As the breakdown spot does not recover from the first emission (caused surface plasma, surface damage, etc.) in such a short time, another breakdown can be triggered in the half cell when the power from the full cell flows in. For multiple-breakdown events, the excitation of the HOM caused by the second breakdown is always near the end of the fundamental mode collapse, as shown in Fig. 3(f). This observation also confirms the second breakdown is triggered by the rf power flow.

\section{Radio frequency breakdown with a laser trigger 1. Experiment observation}

During the laser trigger breakdown experiment, the gradient on the cathode was limited to $48 \mathrm{MV} / \mathrm{m}$. Under this condition, when the laser intensity was very low $(<5 \mu \mathrm{J})$, the normal photoemission process occurred without breakdown. When the laser energy was increased, the breakdown rate also increased. At laser energies greater than $100 \mu \mathrm{J}$, breakdowns occurred at every rf pulse. In order to determine the damage caused by the laser on the cathode, a parallel test was carried out in which the same laser was transported to another copper sample. This sample was subsequently inspected using white light interferometry [25]. For $100 \mu \mathrm{J}$ laser energy, the average intensity is $10 \mathrm{~mJ} / \mathrm{cm}^{2}$ which is an order of magnitude below the ablation threshold $[26,27]$. However, two ablation points were found within the laser induced melting area because of the nonuniform energy distribution.

The same FFT method was also applied to the pickup signal of laser-triggered breakdown events and the result also shows the excitation of the $8.6 \mathrm{GHz}$ HOM. The collapse time of the fundamental mode was $\sim 80 \mathrm{~ns}$, in agreement with the duration of the Faraday cup signal. The onset of the fundamental mode collapse, the appearance of the HOM, and the starting time of the captured breakdown current agree well with each other.

Similarly to those breakdowns occurring without a laser trigger, a laser-triggered breakdown can also be classified into two types according to the breakdown current pulses. However, multiple-breakdown events were quite rare and less than 10 of them were recorded of a total of $1000 \mathrm{rf}$ breakdown events. This is because the breakdown position is at the central area of the cathode and the emitted electrons can pass through two cells and lower the field in them simultaneously. Then less power will flow into the half cell from the full cell; therefore, the possibility of triggering another breakdown is much lower than that without the laser trigger. Figure 4 shows typical waveforms of the two types.

Formation of the laser induced prebreakdown conditions including melting and ablation of copper occurring on the scale of tens of picoseconds [27,28] and the moment of breakdown occurrence varies with the rf phase at which the laser is injected. However, the physical evolution of EEE is
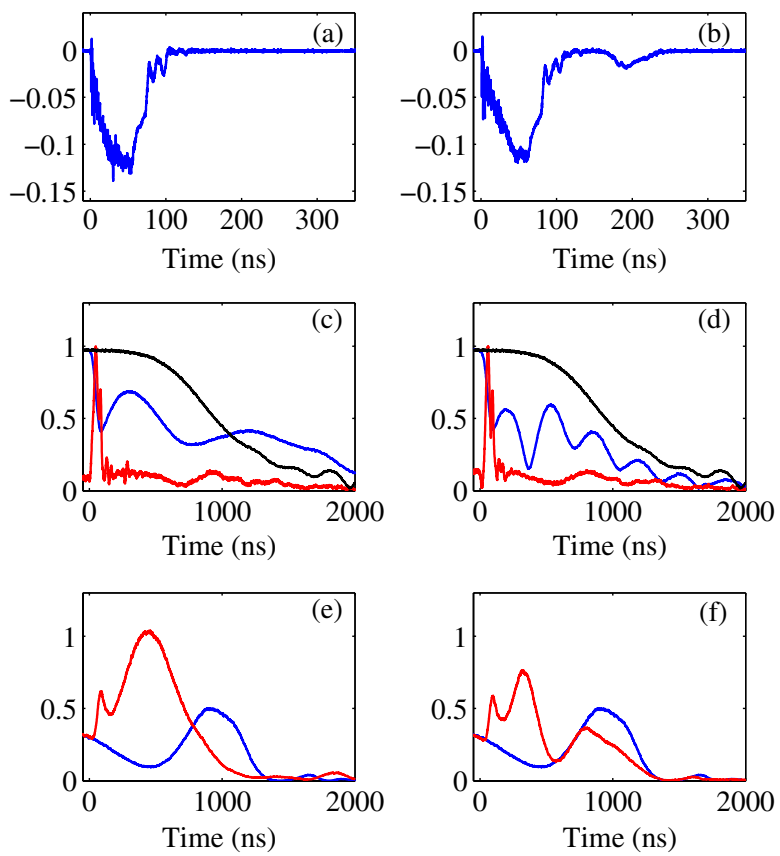

FIG. 4. Radio frequency breakdown with a laser trigger of a single-breakdown event (left) and multiple-breakdown event (right). (a),(b) The Faraday cup signal in amperes. (c),(d) Normalized FFT amplitude of the pickup signal in different frequency bands as a function of time, including the fundamental mode without breakdown (black line), the fundamental mode with breakdown (blue line), and the $8.6 \mathrm{GHz} \mathrm{HOM}$ with breakdown (red line). (e),(f) Normalized FFT amplitude of the reflection signal, including the fundamental mode without breakdown (blue line) and with breakdown (red line).

around a hundred nanoseconds [18] so that the average gradient inside the cavity plays a more important role in the breakdown strength (represented by the charge collected at the exit of the cavity). Figure 5 shows its dependence upon the accelerating gradient.

At a gradient of $48 \mathrm{MV} / \mathrm{m}$, the stored energy in the full cell is $0.9 \mathrm{~J}$ and the average energy gain of a captured electron in the full cell is $\sim 1 \mathrm{MeV}$ (simulation results using PARMELA code [23]). From Fig. 4(c), the pickup signal fell to 0.4 times its original level after the emission occurred. Thus $\sim 0.76 \mathrm{~J}$ is lost during the emission period. Assuming all the lost energy is absorbed by the captured electrons, the upper limit on the emitted charge is $760 \mathrm{nC}$. This estimate ignores the loss of energy gain due to the collapse of the field of the fundamental mode, otherwise the upper limit would be even higher. The captured charge and its upper limit are 2-4 orders of magnitude higher than that of the normal photoelectron $(\sim 100 \mathrm{pC})$.

When a breakdown was triggered by laser, a bright spot could be observed by the CCD camera through the viewport at the half cell, as shown in Fig. 6(a). This bright spot was not observed when only photoelectrons were emitted without rf breakdown. The bright spot position followed the tilting of the UV laser mirror. Figure 6(b) shows the 


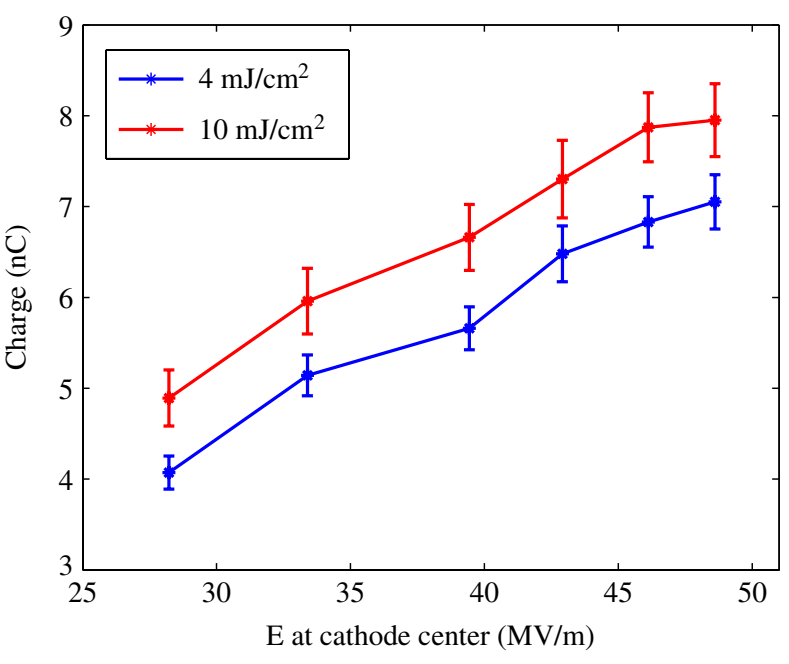

FIG. 5. Captured charge measured at the exit of the rf gun as a function of electric field at the cathode center for two different average laser input energy intensities.

image taken when the light was on; the dark mark at the same place also confirmed the breakdown location. This bright spot together with the high charge indicate the presence of a local plasma during the EEE process near the breakdown spot.

Apart from the $10 \mathrm{~ns}$ window FFT method to study the evolution of fundamental mode and HOMs, another FFT method with a longer window (2000 ns) and thus higher frequency domain resolution $(0.5 \mathrm{MHz})$ was also applied to study the frequency shift of the gun after the onset of rf breakdown. Results show a $1.5 \mathrm{MHz}$ frequency downshift in signal-breakdown events and no frequency shift in multiple-breakdown events. The former case is easy to understand as plasma propagating along the axis (high $E$-field region) detunes two cells to a lower frequency. But the latter case has yet to be understood. It seems that the second breakdown detunes the cells to a higher frequency and hence cancels the frequency downshift by the first breakdown.

\section{Analysis method}

Because of the outer circuit of the Faraday cup, the observed signal is a mixture of a real emission current signal and the oscillations from its intrinsic circuit response

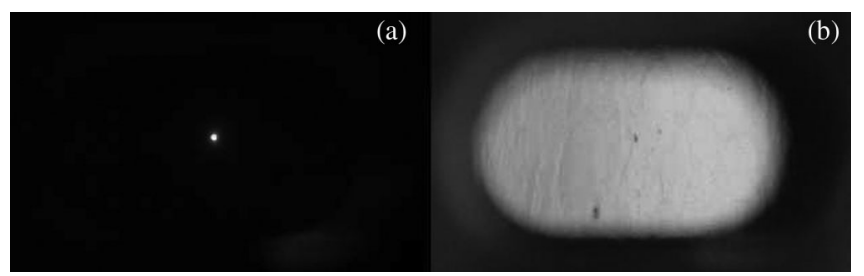

FIG. 6. Cathode images. (a) During a breakdown when light was of.f (b) After breakdown when light was on.
[Fig. 2(c)]. We use a deconvolution method to rebuild the time structure of the breakdown current. The Faraday cup and its outer circuit can be treated as a linear time-invariant system and the detected current can be written as

$$
y(t)=\int_{-\infty}^{+\infty} x(\tau) h(t-\tau) d \tau
$$

where $x(t)$ is the real emission current and $h(t)$ is the impulse response of the system. Because the length of the photoelectron current was only several picoseconds and even shorter than the 20 ps sampling interval of the scope, the detected photoelectron current shown in Fig. 2(c) can serve as the impulse response. Equation (1) can be solved by a deconvolution method [29]. Figure 7 shows the Faraday cup signals before and after deconvolution for both single-breakdown and multiple-breakdown events with laser triggers.

The deconvoluted time structures are slightly different in both events. The formation of a plasma during the EEE process depends on several parameters, such as surface condition, laser intensity, rf field, etc. These conditions fluctuated between every rf pulse. Thus this difference in time structure originates from variation of different pulses rather than different types. They share a few common features. A significant impulse function (the deconvoluted photoelectron current) can be found at the start of the breakdown current. The deconvoluted signals also indicate three stages after the onset of rf breakdown: increasing electron emission (20-50 ns); steady state emission (20-40 ns); and decreasing emission (20-30 ns).

An equivalent circuit model (Fig. 8), where the photocathode gun cavity is modeled as two coupled RLC resonators excited by external rf power [30] is developed to study the evolution of the fundamental mode after the onset of $\mathrm{rf}$ breakdowns. The main parameters of the circuit
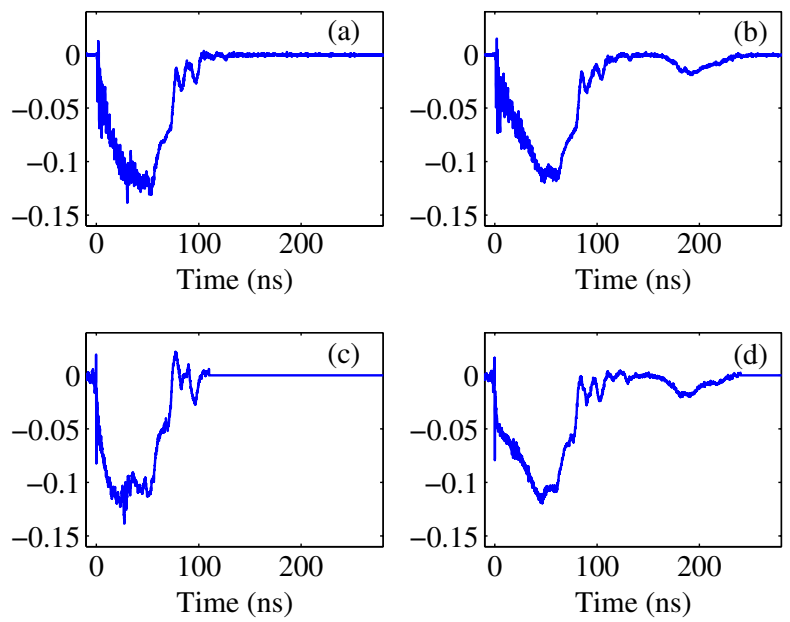

FIG. 7. The Faraday cup signal in amperes of a singlebreakdown event (left) and a multiple-breakdown event (right). (a),(b) Before deconvolution. (c),(d) After deconvolution. 


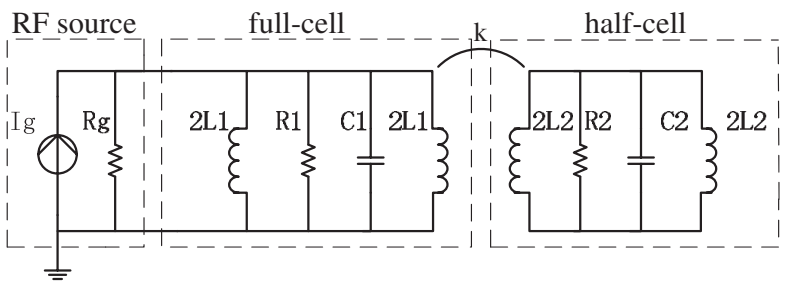

FIG. 8. Equivalent circuit model of the gun.

model under 4.8 MW input power are listed in Table I. Simulation results agreed well with the experimental observation in normal operation, as Fig. 9 shows.

For rf breakdown events, two current sources $I_{1}$ and $I_{2}$ are introduced to the circuit to simulate the explosive emission current, as Fig. 10 shows. The time structure of these currents is the same as the deconvoluted result and their phases are opposite to the corresponding cell voltages.

Based on the FFT results with 2000 ns window, frequency detuning of the two cells is also introduced to the model. The actual plasma proportion and corresponding influence on frequency are complex. In the model, the onset of frequency detuning is chosen based on the speed of plasma movement and best match with the observed rf signal during the breakdown. As the breakdown occurred on the cathode, the half cell is set to be detuned by a certain value immediately after the EEE. In a previous experiment, the highest ion speed $v_{s}$ in the cold explosions was obtained by fitting the data to be $2 \times 10^{4} \mathrm{~m} / \mathrm{s}$ [31]. Theoretical work analyzed the arrival time spectra of the ions and showed that ions can reach even higher speeds when the thermal energy is increased [32]. So the full cell is set to start detuning $200 \mathrm{~ns}$ after the onset of the breakdown and linearly shifted lower to a certain value. When the magnitude of the two currents is $18 \mathrm{~A}$ and the frequency shifts are 3.2 $\mathrm{MHz}$ and $0.9 \mathrm{MHz}$ for the half cell and full cell, respectively, the simulation agrees well with the singlebreakdown experiment result, as shown in Figs. 11(a) and 11(c). The frequency of the $\pi$ mode is then downshifted by 1.7 MHz, which also agrees with the FFT result. The stored energy in the full cell and half cell before the onset of breakdown is $0.91 \mathrm{~J}$ and $0.40 \mathrm{~J}$, respectively. The calculated energy absorbed by the explosive emission current is $0.78 \mathrm{~J}$

TABLE I. Parameters of the circuit model.

\begin{tabular}{lccc}
\hline \hline Parameter & \multicolumn{3}{c}{ Value } \\
\hline$I_{g}(\mathrm{~A})$ & & 5.93 & \\
$k(\%)$ & & 0.22 & \\
$R_{g}(\Omega)$ & & $1.09 \times 10^{6}$ & \\
& & half cell \\
$f(\mathrm{MHz})$ & full cell & & 2854.39 \\
$R(\Omega)$ & 2855.36 & & $4.35 \times 10^{5}$ \\
$C(\mathrm{pF})$ & $1.81 \times 10^{6}$ & & $5.00 \times 10^{-1}$ \\
\hline \hline
\end{tabular}
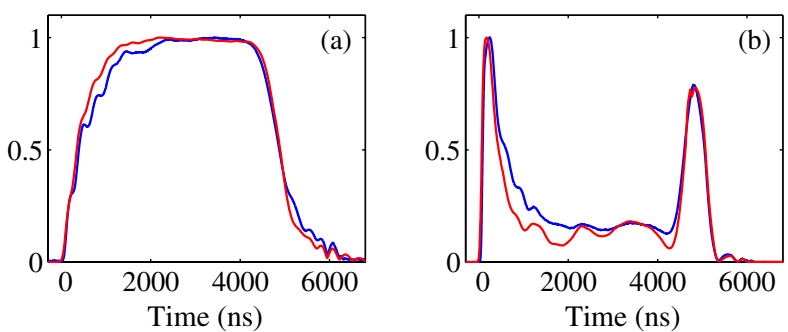

FIG. 9. Comparison of simulation (red line) and experimental results (blue line) without breakdown. (a) Normalized pickup signal. (b) Normalized reflection signal.

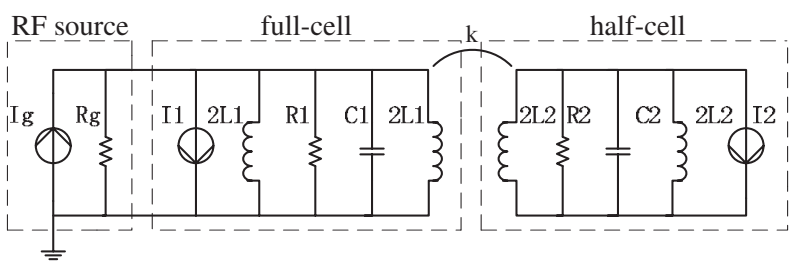

FIG. 10. Equivalent circuit model of the gun with two explosive emission current sources.

in the full cell and $0.39 \mathrm{~J}$ in the half cell. The simulation of multiple-breakdown events is more complex and a second detuning to the half cell after the onset of the second EEE is introduced as discussed before. The upper shift is set to 1.3 MHz and the simulation shows reasonable agreement with experiment, as shown in Figs. 11(b) and 11(d).

In the circuit model, the time delay between the onset of collapse of the fundamental mode and the excitation of
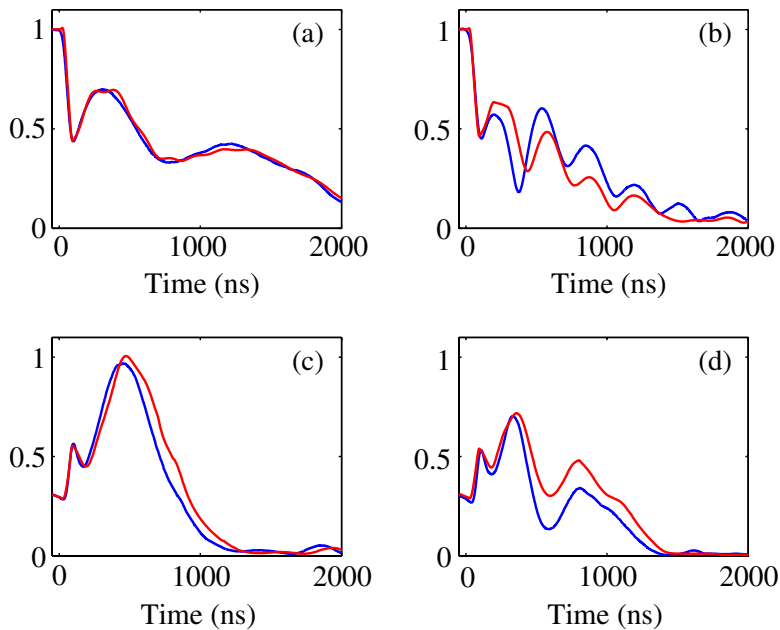

FIG. 11. Comparison of simulation (red line) and experimental results (blue line) of single-breakdown event (left) and multiplebreakdown event (right). (a),(b) Normalized pickup signal. (c), (d) Normalized reflection signal. 
HOM without the laser trigger can be simulated with an assumption that $I_{1}$ is zero for certain time interval.

\section{DISCUSSION}

Similarities in many aspects have been found between $\mathrm{rf}$ breakdowns with and without laser triggers, such as excitation of HOMs, frequency shift of the gun cavity after the onset of breakdown events, two types of breakdown events according to the detected charge pulses, etc.

Meanwhile, there exist some noticeable differences between rf breakdowns with and without the laser trigger. For breakdowns without the laser trigger, the shape of the current is spiky and the captured charge is 2 orders of magnitude lower than that with the laser trigger. Besides, the captured charge with the laser trigger shown in Fig. 5 has a small variance at a certain field and laser intensity. While for the data without the laser trigger, the variance is too large to give any statistically significant results on the properties of breakdown. These phenomena are consistent with our analysis of breakdown locations.

We understand that in the experiment the intense laser assisted the plasma formation on the cathode surface at a time and location corresponding to the presence of a large electric field which caused the breakdown to occur. It suggests for rf breakdowns that the limit in high gradient operation in this photocathode gun may occur predominately in a high electric field region at the surface. In future experiments, the laser could also be directed to a high magnetic field or high power rf flow region in other photoinjectors to study additional if breakdown phenomena in a more controllable way.

Laser triggering is also a more flexible method as the breakdown rate can be very high with high laser intensities. Thus much time can be saved in obtaining a large amount of breakdown data and some quick experiments will be able to be carried out then.

Generally speaking, when a breakdown occurs in a traveling wave structure, the power flow will be absorbed inside the structure, which causes severe damage. While for standing wave structures, the input power will be reflected and thus will cause less damage. Previous studies have found that standing wave structures operating at $\pi$ mode have a lower breakdown rate at a given gradient compared with traveling wave structures [33]. In the experiment, we observed multiple breakdowns within one rf pulse caused by the power flow from another cell into the cell where the first breakdown occurred. This observation implies in multiple-cell standing wave structures, subsequent breakdowns within one rf pulse can be triggered after the first one even when the input power is reflected. To avoid this phenomenon and its damage, the cells need to be separated from each other. Using an individually rf feeding method $[33,34]$, it is possible to design such a standing wave structure with zero coupling between cells.

\section{SUMMARY}

In summary, it is demonstrated that rf breakdown in the photocathode gun can be triggered by an intense laser pulse at predictable positions and times. The time evolution of catastrophic electron emission at the nanosecond scale after the onset of breakdown has been observed and analyzed. Comparison between rf breakdowns with and without laser triggering shows that the laser trigger can serve as a more controllable and flexible method for breakdown study. The experiment reveals that multiple breakdowns can be triggered by the power flow between cells after the initial EEE and implies standing wave structures with separated cells may be favorable for high gradient accelerators. Subsequent experiments are planned at the Argonne Wakefield Accelerator facility.

\section{ACKNOWLEDGMENTS}

We would like to thank Jin Yang, Dan Wang and Zhen Zhang from Tsinghua University and Sergey V. Baryshev from Euclid Techlabs LLC for their support during the experiment. The work at Tsinghua University is supported by National Natural Science Foundation of China under Grant No. 11135004. The work by Faya Wang is funded through the U.S. Department of Energy Early Career Research Program under project code LAB 11-572. The work by Wei Gai and Chunguang Jing is funded through the U.S. Department of Energy Office of Science under Contract No. DE-AC02-06CH11357.

[1] J. W. Wang and G. A. Loew, SLAC PUB 7684, 1997.

[2] A. Grudiev, S. Calatroni, and W. Wuensch, Phys. Rev. ST Accel. Beams 12, 102001 (2009).

[3] D. P. Pritzkau, Ph.D. thesis, Stanford University, 2001.

[4] V. A. Dolgashev and S. G. Tantawi, SLAC PUB 10175 2003.

[5] K. Nordlund and F. Djurabekova, Phys. Rev. ST Accel. Beams 15, 071002 (2012).

[6] T. Higo et al., in Proceeding of the 2012 Linear Collider Workshop, Arlington, TX, 2912.

[7] V. Dolgashev et al., in Proceedings of the 2013 High Gradient Workshop, ICTP, Italy, 2013.

[8] B. Munroe, A. Cook, M. Shapiro, R. Temkin, V. Dolgashev, L. Laurent, J. Lewandowski, A. Yeremian, S. Tantawi, and R. Marsh, Phys. Rev. ST Accel. Beams 16, 012005 (2013).

[9] F. Wang, C. Adolphsen, and C. Nantista, Phys. Rev. ST Accel. Beams 14, 010401 (2011).

[10] F. Wang, C. Adolphsen, and C. Nantista, in Proceedings of the 24th Particle Accelerator Conference, PAC-2011, New York, 2011 (IEEE, New York, 2011), TUP139.

[11] A. D. Yeremian, V. A. Dolgashev, J. Nielson, and S. G. Tantawi, in Proceedings of the 2nd International Particle Accelerator Conference, San Sebastián, Spain (EPS-AG, Spain, 2011), MOPC073. 
[12] A. Descoeudres, Y. Levinsen, S. Calatroni, M. Taborelli, and W. Wuensch, Phys. Rev. ST Accel. Beams 12, 092001 (2009).

[13] H. Timko, M. Aicheler, P. Alknes, S. Calatroni, A. Oltedal, A. Toerklep, M. Taborelli, W. Wuensch, F. Djurabekova, and K. Nordlund, Phys. Rev. ST Accel. Beams 14, 101003 (2011).

[14] CLIC Conceptual Design Report, CERN Technical Report No. CERN-2012-007, 2012.

[15] S. P. Bugaev, E. A. Litvinov, G. A. Mesyats, and D. I. Proskurovskŭ, Sov. Phys. Usp. 18, 51 (1975).

[16] J. W. Wang, Ph.D. thesis, Stanford University, 1989.

[17] X. J. Wang, T. Tsang, H. Kirk, T. SrinivasanRao, J. Fischer, K. Batchelor, P. Russell, and R. C. Fernow, J. Appl. Phys. 72, 888 (1992).

[18] E. A. Litvinov, G. A. Mesyats, and D. I. Proskurovskŭ, Sov. Phys. Usp. 26, 138 (1983).

[19] Y.E. Krasik, A. Dunaevsky, and J. Felsteiner, Phys. Plasmas 8, 2466 (2001).

[20] A. V. Gunin, A. I. Klimov, S. D. Korovin, I. K. Kurkan, I. V. Pegel, S. D. Polevin, A. M. Roitman, V. V. Rostov, A. S. Stepchenko, and E. M. Totmeninov, IEEE Trans. Plasma Sci. 26, 326 (1998).

[21] W. Wuensch, in Proceedings of the 8th European Particle Accelerator Conference, Paris, 2002 (EPS-IGA and CERN, Geneva, 2002), MOYGB003.

[22] R. Li, C. Tang, Y. Du, W. Huang, Q. Du, J. Shi, L. Yan, and X. Wang, Rev. Sci. Instrum. 80, 083303 (2009).
[23] L. M. Young, PARMELA, Los Alamos National Laboratory Technical Report No. LA-UR-96-1835, 1996.

[24] CST Microwave Studio, CST, Bad Nauheimer Str. 19, 64289 Darmstadt, Germany.

[25] S. V. Baryshev, R. A. Erck, J. F. Moore, A. V. Zinovev, C. E. Tripa, and I. V. Veryovkin, J. Vis. Exp. 72, e50260 (2013).

[26] S. Preuss, A. Demchuk, and M. Stuke, Appl. Phys. A 61, 33 (1995).

[27] C. Schäfer, H. M. Urbassek, and L. V. Zhigilei, Phys. Rev. B 66, 115404 (2002).

[28] H. Häkkinen and U. Landman, Phys. Rev. Lett. 71, 1023 (1993).

[29] B. Drachman, IEEE Trans. Antennas Propag. 32, 219 (1984).

[30] F. Wang and C. Adolphsen, Phys. Rev. ST Accel. Beams 12, 042001 (2009).

[31] M. Johnson, R. Ruber, V. Ziemann, and H. Braun, Nucl. Instrum. Methods Phys. Res., Sect. A 595, 568 (2008).

[32] V. Ziemann, Nucl. Instrum. Methods Phys. Res., Sect. A 575, 539 (2007).

[33] J. Neilson, S. Tantawi, and V. Dolgashev, in Proceeding of the 5th Collaboration Meeting on X-band Accelerator Structure Design and Test Program, Menlo Park, CA, 2011.

[34] S. G. Tantawi, Phys. Rev. ST Accel. Beams 9, 112001 (2006). 